\title{
Spectrally Resolved Fluorescence Correlation Spectroscopy Based on Global Analysis
}

\author{
Michael J. R. Previte ${ }^{\star}, \dagger$, , Serge Pelet ${ }^{\dagger}$, , Ki Hean Kim $^{\dagger, \|}$, Christoph Buehler $^{\dagger, \perp}$, and Peter T. \\ C. So ${ }^{\dagger, \#}$
}

tDepartment of Biological Engineering, Massachusetts Institute of Technology, 77 Massachusetts

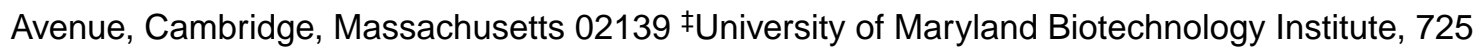
West Lombard Street, Baltimore, Maryland 21202 \& Institute of Biochemistry Swiss Federal Institute of Technology Zurich (ETH), Schafmattstrasse 18 HPM G9.1, CH-8093 Zurich, Switzerland "Wellman Center for Photomedicine, Massachusetts General Hospital, 50 Blossom Street, BAR714, Boston, Massachusetts $02114{ }^{\perp}$ Novartis Institutes for BioMedical Research, Vienna, Innovative Screening Technologies, Brunner Strasse 59, A-1235 Vienna, Austria \#Department of Mechanical Engineering, Massachusetts Institute of Technology, 77 Massachusetts Avenue, Cambridge, Massachusetts 02139

\section{Abstract}

\begin{abstract}
Multicolor fluorescence correlation spectroscopy has been recently developed to study chemical interactions of multiple chemical species labeled with spectrally distinct fluorophores. In the presence of spectral overlap, there exists a lower detectability limit for reaction products with multicolor fluorophores. In addition, the ability to separate bound product from reactants allows thermodynamic properties such as dissociation constants to be measured for chemical reactions. In this report, we utilize a spectrally resolved two-photon microscope with single-photon counting sensitivity to acquire spectral and temporal information from multiple chemical species. Further, we have developed a global fitting analysis algorithm that simultaneously analyzes all distinct auto- and cross-correlation functions from 15 independent spectral channels. We have demonstrated that the global analysis approach allows the concentration and diffusion coefficients of fluorescent particles to be resolved despite the presence of overlapping emission spectra.
\end{abstract}

Fluorescence correlation spectroscopy (FCS) was developed to study the temporal correlations of thermodynamic concentration fluctuations in a reactive chemical system and was described in a series of papers by Magde, Webb, and Elson. ${ }^{1,2}$ FCS monitors temporal variations of the fluorescence signal in an optically defined focal volume. These intensity fluctuations originate from important dynamic properties, such as diffusion, aggregation, chemical and enzyme kinetics, flow, and active transport. ${ }^{1-3}$ The amplitude of these fluctuations is proportional to the square root of the number of molecules in the volume. 3,4

\footnotetext{
*To whom correspondence should be addressed.prev_31k@mit.edu. 
Fluorescence cross-correlation spectroscopy (FCCS) can also be a powerful tool to study intracellular signaling processes regulated by the binding of protein species and complex formation. ${ }^{5}$ The concentrations of individual species labeled with spectrally distinct fluorescent tags and the kinetics of any subsequent complex formation have been successfully resolved with FCCS using either one or two excitation sources. ${ }^{6-8}$ Due to the stringent alignment requirements in combining two excitation sources, the use of two-photon excitation overcame these technical disadvantages and facilitated the excitation of multicolor fluorescent mixtures. ${ }^{9,10}$ For example, two-photon excitation of up to three dyes has been implemented to simultaneously resolve molecular concentrations and kinetics of three distinctly labeled, interacting species. ${ }^{8,11}$ More recently, one-photon excitation has been implemented for FCCS studies, whereby a single laser line has been used to excite two fluorophores with similar excitation but different emission characteristics. ${ }^{12}$ Complexes formed from the reactants labeled with different color fluorophores results in a new spectrally distinct species with a correlation trace that can be fit to quantify the kinetics, dynamics, and concentration of this complex. ${ }^{6,9,13}$ Although the cross-correlation signal is specific to doubly labeled (or triply labeled) species, the accurate recovery of the dynamics and concentrations of the complex is critically dependent on the emission spectra of the labels. 5,14

For fluorescent labels that do not have cleanly separated spectra, the reliability of multicolor FCCS methodologies becomes limited as a result of spectral cross talk. ${ }^{5,14}$ Schwille and coworkers also described that the extent of spectral cross talk is not only influenced by the spectral properties of the respective labels but it also depends on the choice of concentration and brightness ratios of the dyes. In many instances, the inappropriate choice of fluorescent labels leads to inaccurate measurements of correlation amplitudes. Although many new genetically expressible fluorescent proteins have been introduced as candidates for multicolor FCS applications in living cells, ${ }^{15}$ the application of multicolor FCS with genetically expressible probes remains limited due to many inherent complications of an intracellular experiment, such as variable expression levels, brightness, spectral overlap, and background autofluorescence. ${ }^{15}$ Although this technique is promising, it remains limited due to the number of fluorophores that are available with significant Stokes shifts to be resolved with FCCS. As a result, these variables introduce significant complexity in data analysis and continue to limit the broad applicability of FCS to study real-time signaling processes in living cells.

In order to address the issue of spectral overlap, most multicolor FCS studies use fluorescent species with well-resolved spectra to minimize the issue of degrading FCCS signals. ${ }^{6-8,16}$ In addition, dichroics and barrier filters are carefully chosen to maximize signal and minimize cross talk to alleviate the degradation of FCCS signals. ${ }^{5}$ Thus, there exists a lower limit for detecting reaction complexes based on multicolor cross-correlation analysis and other analysis approaches such as photon counting histogram and moment analysis. ${ }^{17,18}$

Potential solutions to address the issue of spectral cross talk have been proposed to resolve number concentrations of multicolor complexes. For two and three color analyses, coincidence analysis has proven successful in retrieving parameters of interest. ${ }^{7,8,11}$ Pulsed interleaved excitation, which implements an alternating excitation scheme at a rate faster 
than the diffusion times of the components, has also been implemented to resolve multicolor mixtures, ${ }^{19-21}$ A Bayesian estimation approach was theoretically described to determine both diffusion coefficients and concentrations for multicolor solutions, but experimental results have yet to be shown. ${ }^{22}$ Various spectral imaging methods have also been used to resolve protein species labeled with spectrally overlapping probes. ${ }^{23-25}$ Due to slow data acquisition time and noisy analog detection approaches, they have had limited applications with FCS. Multicolor FCS with up to four different channels was recently described, but is precluded by species with overlapping spectra, and simultaneous photon counting for all spectral channels was not possible. ${ }^{16}$

Global analysis of FCS data has been reported to be an effective tool to accurately recover concentrations and diffusion times of species in solution..$^{26,27}$ The combination of global analysis of FCS data and FRET (2CG-FCS) has been reported to accurately recover the diffusion properties, molecular concentration, and biomolecular interactions for two color fluorescent mixtures. ${ }^{28}$ While 2 CG-FCS accurately retrieves the characteristics of two color FRET mixtures, the report provides evidence that global fitting can also be successfully implemented to resolve mixtures of fluorescent species with significant channel cross talk.

Complementary to the developments of multichannel (>3) $\mathrm{FCS}^{8,16,29}$ and 2CG-FCS, ${ }^{28}$ we introduce in this report, a strategy to further resolve complex fluorescent mixtures. We combine the benefits of spectrally resolved microscopy (temporal data from 15 spectral channels acquired simultaneously) with global fitting analysis of FCS data. With spectrally resolved fluorescence correlation spectroscopy with global analysis (SRFCS-G), we resolve the diffusion properties and molecular concentration of individual fluorescent components from multicomponent mixtures of fluorescent species with significant spectral overlap.

\section{EXPERIMENTAL METHODS}

\section{Samples}

We sought to recover the diffusion coefficients and concentrations of fluorescent probe combinations previously unresolved with multicolor FCS studies. We demonstrate our technique using fluorescent bead mixtures. Bead mixtures were prepared in HPLC pure water (Sigma-Aldrich) from filter-purified (Pall, $0.2-\mu \mathrm{m}$ syringe filter) 20 -nm yellow-green ( $\mathrm{G}, 505 / 515)$, orange $(\mathrm{O}, 535 / 575)$, and red $(\mathrm{R}, 580,675)$ polystyrene beads (Molecular Probes, Eugene, OR) and imaged in eight chamber no. 1.5 cover glass slides (Lab-TekII, 155409). From the stock bead solutions, we created two color or three color mixtures of yellow-green/red (G/R) and yellow-green/orange (G/O) or yellow-green/red/orange beads. The expected bead ratios were calculated based on bead dilutions and the stock solution concentrations. As reference for some of our measurements, we have used a 50\% glycerol solution containing $25 \mathrm{nM}$ Alexa Fluor 488 (Molecular Probes)

\section{Instrumentation}

The multicolor FCS system is developed by combining a two-photon microscope, a grating spectrograph (SG), a multianode PMT, and a multichannel single-photon counting electronics (MCPC, Figure 1). A 63×, 1.2 NA objective (C-apochromat, Zeiss Inc., 
Thornwood, NY) is used to produce a near-femtoliter focal volume. Samples were excited using a femtosecond laser (Mira, Coherent, Santa Clara, CA) generating 100-fs pulses with a center wavelength of $780 \mathrm{~nm}$ at $80 \mathrm{MHz}$. In order to minimize photobleaching during data collection, typical excitation power was $5 \mathrm{~mW}$ at the sample. The spectrograph, multianode PMT, and MCPC is configured to simultaneously collect photons from 15 spectrally resolved channels with a spectral range from 440 to $655 \mathrm{~nm}$ at a resolution of $15 \mathrm{~nm}$. Singlephoton counting electronics provide sustained transfer to the computer memory from all 15 channels simultaneously up to a rate of $250 \mathrm{kHz}{ }^{30}$ This spectrally resolved FCS has comparably lower photon detection sensitivity as compared to a conventional single-channel FCS system using a PMT due to the descanning optics ( $\sim 60 \%$ loss), the grating ( $\sim 67 \%$ loss), and the less sensitive multianode PMT. Signal loss at the red edge of the spectrum is particularly severe where PMT quantum efficiency decreases. The overall photon detection yield is $\sim 3-5 \%$ efficiency for channels with maximum quantum efficiency, but less than $1 \%$ for red wavelengths. On board memory restriction allows data acquisition for up to $30 \mathrm{~s}$; multiple data sets were collected for low signal-to-noise specimens.

\section{Data Analysis}

FCS fitting algorithms for traces of independent freely diffusing species in a 3D Gaussian observation volume use the following equation: ${ }^{6}$

$$
\begin{aligned}
& G_{i i, k}(\tau)=\left(\pi^{3 / 2} r_{\mathrm{o}}{ }^{2} z_{\mathrm{o}}\right)^{-1}\left\langle C_{k}\right\rangle^{-1} \operatorname{Diff}_{k} \\
& \operatorname{Diff}_{k}=\left(1+\frac{\tau}{\tau_{\mathrm{D}, k}}\right)^{-1}\left(1+c \frac{\tau}{\tau_{\mathrm{D}, k}}\right)^{-1 / 2}
\end{aligned}
$$

where $i$ is the spectral channel for the emission of species $k, V_{\text {eff }}$ is the two-photon effective focal volume where $V_{\text {eff }}=\pi^{3 / 2} r_{\mathrm{o}}^{2} z_{\mathrm{o}}$ and $r_{\mathrm{O}}$ and $z_{\mathrm{O}}$ are the lateral and axial $1 / e^{2}$ distances for a theoretical Gaussian emission light distribution, $c$ is the aspect ratio $\left(r_{0}^{2} z_{\mathrm{O}}{ }^{-2}\right), \tau$ is the delay time, and $\tau_{\mathrm{D}, k}$ is the average diffusion time for species $k$, where $\tau_{\mathrm{D}, k}=r_{o}^{2}\left(8 D_{k}\right)^{-1}$ for a twophoton process and $D_{k}$ is the diffusion constant for species $k$. Calibration factors, such as effective volume ( $V_{\text {eff }} \approx 1 \mathrm{fL}$ ), and the aspect ratio, $c$, were determined using a 50\% glycerol solution containing $25 \mathrm{nM}$ Alexa 488 and data that were summed across all 15 spectral channels. Along with the geometrical parameters cited above, the molecular brightness of the Alexa 488 dye for the spectral detection scheme was found to be $\sim 1.7 \mathrm{kHz} /$ molecule integrated over all spectral channels. The brightness for an individual channel is consistent with the contribution calculated from linear spectral decomposition. While preliminary studies with molecular detection proved to be difficult, polystyrene fluorescent spheres are $\sim 150$ times brighter than the fluorescent dye. As a result, we could readily resolve polystyrene bead dynamics and number concentrations with this configuration. 
In order to simulate a two-channel (or three-channel) detection scheme, we binned spectral data that corresponded to a 60- or 75-nm window for each independent species. For example, green and red channels for two-color green and red mixtures were created by binning photon counts for channels 5-9 and channels 10-15, respectively. These binned data represent the conventional two-channel or three-channel detection scheme. Each of the two or three individual channel data was independently correlated and fit to eq 1 to extract the diffusion time $\left(\tau_{\mathrm{D} k}\right)$ and the average number density $\left(C_{k}\right)$ of that compound.

Global analysis across interdependent data sets allows an improved recovery of fitting parameters by putting forward common features present in all the data sets and minimizing their individual noise. ${ }^{31}$ Global analysis has been used extensively in fluorescence lifetime analysis, ${ }^{32,33}$ but its application in FCS analysis has been rare. ${ }^{26,28,34}$ The auto- and crosscorrelation functions across the 15 spectral channels can be expressed as

$$
G_{i j}(\tau)=\left(\sum_{k=1}^{R} \eta_{k}{ }^{2} S_{i k} S_{j k}\left\langle C_{k}\right\rangle \operatorname{Diff}_{k}\right) \times\left[V_{e f f}\left(\sum_{k=1}^{R} \eta_{k} S_{i k}\left\langle C_{k}\right\rangle\right)\left(\sum_{k=1}^{R} \eta_{k} S_{j k}\left\langle C_{k}\right\rangle\right)\right]^{-1}
$$

where $i$ and $j$ refer to the spectral channel number, $\eta_{k}$ is the brightness of species $k,\left\langle C_{k}\right\rangle$ is the average number density of species $k, S_{i, k}$ is spectral component $i$ for species $k$, and $S_{j, k}$ is spectral component $j$ for species $k$. Reference spectra and brightness parameters for each independent bead species were determined and fixed prior to global fitting. Diffusion constants and number density for each species were globally fit according to eq 3 using a minimization algorithm based on the Levenberg-Marquardt method. ${ }^{35}$

For all of the bead mixtures, we recorded 10 data sets at 5 - $\mu$ s time intervals for $\sim 30 \mathrm{~s}$. Photon counts were collected in the 15 detection channels covering the emission spectra of the fluorescent species studied. For 8-channel detection, 2 consecutive spectral channels were binned to generate 7-channel data, and the eighth channel represented of the counts collected by channel 15 . Auto- and cross- correlation curves were calculated across all 15 (or 8) spectral channels. (Figure 5A-C and SI Figure 3A-C).

We also performed a global fit including spectral constraints. For this fitting algorithm, we integrated an additional fitting function, $\sum_{k=1}^{R} \eta_{k}{ }^{2} S_{i k} S_{j k}\left\langle C_{k}\right\rangle$, to further constrain the global fit (represented as $15 \mathrm{CH}$-spec or $8 \mathrm{CH}$-spec in Figure 6). With this additional constraint, we hoped to more accurately recover concentrations and diffusion times and minimize fitting times.

\section{RESULTS}

\section{Single Species}

Due to the on board memory restrictions, the minimum time resolution that was achievable with this configuration was $\sim 5 \mu \mathrm{s}$. To calibrate the focal volume and aspect ratio with a known dye concentration, we used 50\% glycerol A488 solutions to decrease the diffusion times of the dye by a factor of $\sim 5$, which was within the time resolution of our 
instrumentation. We took 10 consecutive data sets at 5 - $\mu$ s time intervals for $\sim 30$ s for a $50 \%$ glycerol solution containing $25 \mathrm{nM}$ Alexa 488. Subsequently, we compared the autocorrelation curves of channels 3-6 with the sum of the fluorescence fluctuations across all channels (Figure 2A). Single-channel fits were performed for correlation curves representative of individual and summed channel data. For the correlation curves from the summed and individual data, we recovered comparable diffusion times and numbers of molecules (Figure 2A, solid line and Supporting Information (SI) Table 1). From these results, we determined that the correlation curves for multicolor fluorescent solutions are not affected by instrumental correlation artifacts.

Normalized intensity spectra for green, orange, and red 20-nm-diameter beads are plotted versus wavelength and corresponding channel number (Figure $2 \mathrm{~B}$ ). The fluorescence fluctuations for the stock solutions containing single species of $\mathrm{G}, \mathrm{R}$, and $\mathrm{O}$ polystyrene beads are summed across all of the detection channels, and the summed intensity counts are correlated to retrieve a single autocorrelation curve for each individual species (Figure 2C). Since the bead solutions were filter purified to remove the free dye and used as the stock solutions for subsequent experiments, we use the fit from these autocorrelation curves to recover the bead concentrations in the stock solutions. We recovered similar diffusion times for each of these bead solutions, which we expect for equivalent bead diameters ( $\mathrm{G} \sim 2.8 \mathrm{~ms}$, $\mathrm{R} \sim 2.8 \mathrm{~ms}$, and $\mathrm{O} \sim 3.9 \mathrm{~ms}$ ).

\section{Two Color Mixture}

For well-separated spectra (G and R), SRFCS with global analysis (Figure 3A) accurately reproduce results obtained using least-squares fitting of the autocorrelation curves from binned two-channel data. We do not observe any further improvement in the recovered G/R ratios using spectral fitting for species with well-separated spectra. For two species with overlapping spectra, SRFCS with global analysis improves the accuracy of recovered G/O ratios (Figure 3B) versus individually fitting the autocorrelation curves from the individually binned two-channel data. While the photon counting statistics improved for the binned 8channel data, the accuracy of the recovered G/O ratios for the binned 8-channel data is similar to the recovered ratios from the 15-channel data.

Individual fits of the autocorrelation curves from the individually binned two-channel data (eq 1) recovered diffusion times that closely approximate expected results for the $G$ species (SI Figure 1A). We observed that the global fitting approach similarly recovers accurate diffusion times for the $\mathrm{G}$ species, but the recovered diffusion times for the red beads have larger deviations from the expected results (SI Figure 1B). We plotted the recovered diffusion times versus $\eta_{X} N_{X}$, where $\eta_{X}$ is the brightness of species $X\left(\mathrm{G}, \mathrm{O}\right.$, or R) and $N_{X}$, the number of molecules of species $x$. From these results, we observed that the accuracy of the recovered diffusion times of the $G$ species for the $G / R$ mixtures does not dependent on the number of counts per second of the sample measured by the product $\eta_{X} N_{X}$. With regard to the recovered $\mathrm{G}$ diffusion times for the 8-channel data and 15-channel data (SI Figure $1 \mathrm{~A}, \mathrm{~B})$, we observed that the recovered diffusion times are not as accurate as the diffusion times retrieved using the conventional two-channel autocorrelation fitting algorithm. We attribute the overall decreased accuracy of the recovered diffusion times 8- and 15-channel 
data to the decreased photon counts $\left(\eta_{X} N_{X}\right)$ in the individual spectral channels and subsequent noisier correlation curves. Unlike species with non-overlapping spectra, SRFCS with global analysis of the $\mathrm{G} / \mathrm{O}$ mixtures recovered diffusion times for the $\mathrm{G}$ and $\mathrm{O}$ species that were more consistent with the expected results (SI Figure 1C,D) than a conventional fit of autocorrelation curves. Although each of the methods recovered the $\mathrm{G}$ diffusion times that closely matched the expected value, the 15-channel SRFCS with global fitting was the most consistent. For the $\mathrm{O}$ species, the 15-channel SRFCS with global fitting approach also accurately recovered the $\mathrm{O}$ diffusion times (SI Figure 1D).

The results also revealed that the inclusion of spectral constraints on the fitting algorithm did not improve the accuracy of recovered species ratios for the two-color data sets (data not shown). We also did not observe any improvement in the recovery of diffusion times for the $\mathrm{G} / \mathrm{R}$ and G/O mixtures between the analysis models with and without spectral constraints.

\section{Three-Color Mixture}

SRFCS with global fitting achieved much improved accuracy in the recovery of the species ratios for the $\mathrm{G}, \mathrm{O}$, and $\mathrm{R}$ mixtures compared to a three-channel detection scheme. The most accurate recovery of the G/R ratio was obtained using the 15-channel SRFCS and global fitting approach, but the binned 8-channel data with global fitting also performed well (Figure 4A). However, significant error in the recovered $\mathrm{G} / \mathrm{R}$ ratios was measured when we fit the individual autocorrelation curves representing a conventional three-channel detection scheme. Since the ratio of $\mathrm{G}$ and $\mathrm{O}$ species remained constant as the concentration of the $\mathrm{R}$ species varied, the $\mathrm{G} / \mathrm{O}$ ratio was plotted versus the dilution factor. With increasing dilution of the G/O species, we did not observe a loss in accuracy of the recovered ratios with either the 8-channel or 15-channel SRFCS-G method, while the 3-channel detection performs poorly (Figure 4B). The benefits of the SRFCS with global fitting approach are best demonstrated for the recovered ratio of the beads with the largest degree of spectral overlap, $\mathrm{O}$ and R. Using the 8- and 15-channel SRFCS with global analysis, we dramatically improved the recovery of the $\mathrm{O} / \mathrm{R}$ ratios for the three-color mixture compared to the threechannel detection, which shows significant deviation from the expected ratios (Figure 4C). Including spectral constraints in the fitting algorithm did not yield a decrease in fitting times or improved recovery ratios.

For three-color mixtures, we observed that fitting individual autocorrelation curves for a conventional three-channel detection scheme closely approximated expected diffusion time for the $\mathrm{G}$ species, but significantly deviated from the expected value for both the $\mathrm{O}$ and $\mathrm{R}$ species (SI Figure 2AC-). On the other hand, we observed that the 8-channel and 15-channel SRFCS with global fitting satisfactorily recovered diffusion times for both the $\mathrm{G}$ and $\mathrm{R}$ species (SI Figure 2A and C), and, accuracy is not noticeably dependent on $\eta_{X} N_{X}$ (counts/s). The recovered diffusion times for the $\mathrm{O}$ species were however more variable and none of the fitting approaches were able to accurately recover the diffusion time, although fitting the autocorrelation curves for the single $\mathrm{O}$ detection channel proved to be more accurate (SI Figure 2B).

We use contour plots to display raw and fitted data of three-color bead mixtures with green, orange, and red beads with number concentrations of 1.2, 0.8, and 10.3 (Figure 5) and 3.8, 
2.5, and 6.7 (SI Figure 3), respectively. The bottom half of the $15 \times 15$ symmetric matrix and the main diagonal give rise to 120 correlation terms. These correlation terms are represented on the $y$-axis of these plots in an incremental fashion (1-1, 2-1, 2-2, 3-1, 3-2, 3-3, etc.). Raw and fitted correlation decay curves for channels 1-5 (Figure 5A,D), 6-10 (Figure 5B,E), and 11-15 (Figure 5C,F) are shown. These 120 correlation curves were fit globally to obtain a single diffusion time $\left(\tau_{\mathrm{D} k}\right)$ and average number density $\left(C_{k}\right)$ for each species in the mixture.

From the contour plots that correlated with the spectral peaks of the respective species, we observed that the number densities, $\left\langle C_{k}\right\rangle=1 / \mathrm{G}(0)$, and expected diffusion times of the $\mathrm{G}, \mathrm{R}$, and $\mathrm{O}$ species could be estimated by visual inspection. The fit contour plots of three-color bead mixtures that contain an 1.2:0.8:10 ratio of $\mathrm{G}, \mathrm{O}$, and $\mathrm{R}$ species, respectively, are displayed in Figure 5D for channels 1-5, Figure 5E for channels 6-10, and Figure 5F for channels 11-15 and demonstrate the accurate fitting of the cross-correlation raw data (Figure 5A-C) using $15 \mathrm{CH}$ SRCS with global algorithm. For comparison, we also show the contour plots of the raw and fitted results for a triple mixture that is likely more difficult to resolve, due to the number concentrations of the respective species (G, 3.8; O, 2.7; R, 6.5) (SI Figure 3). Again, the contour plots of the fitted data accurately reflect the contour plots of the raw data. In addition, we show the contour plots of the fitted results for a solution containing only green bead species with number concentrations of 8.6. The fitted results accurately reflect a $100 \%$ cross-correlation across these spectral channels, which is the expected result for one diffusing species (SI Figure 4).

In order to compare the overall effectiveness of the respective analysis methods, the deviations of the species ratios for the mixtures are calculated according to the following:

$$
\sigma_{\text {total }}=\sum_{n} \sqrt{\left(\left\langle R_{n, \text { rce }}\right\rangle-\left\langle R_{n, \exp }\right\rangle\right)^{2}}
$$

where $\left\langle R_{n, \text { rec }}\right\rangle$ is the recovered mixture ratios or dilutions, $\left\langle R_{n, \text { exp }}\right\rangle$ is the expected number for the same ratios or dilutions, and $n$ is the number of mixtures.

Overall, the deviations of the number densities for each mixture in the two-color and threecolor experiments were compared to evaluate the general effectiveness of the respective data analysis methods. We observed that the SRFCS with global fitting method outperforms the two- or three-channel detection schemes for recovery of species with overlapping spectra (Figure 6). We also observed significantly improved recovery of the number concentrations for species with overlapping spectra by using 15 detection channels instead of 8 despite the decreased photon counts in each channel. Inclusion of the spectral constraints for both the 8 $\mathrm{CH}$ and $15 \mathrm{CH}$ did not yield any improvement in the recovery of number densities for the species with overlapping spectra.

\section{DISCUSSION}

In this work, we demonstrate that SRFCS with global fitting can effectively recover number concentrations and diffusion times for overlapping species. When compared with an FCS setup with two independent channels, both two-channel detection with autocorrelation fits 
and SRFCS with global fitting effectively recover the expected number concentrations (Figure 3A) and diffusion times (SI Figure 1A,B) for species with non-overlapping spectra. On the other hand, for two-color species with significant spectral overlap, we have shown that SRFCS with global fitting improves the accuracy of recovery for number concentrations (Figure 3B) although the accuracies of the recovery of the diffusion coefficients with SRFCS-G are less accurate (SI Figure 1C,D).

In general, the diffusion time of the $\mathrm{G}$ species can be accurately recovered in either mixture. For the minimal spectral overlap case, the $\mathrm{R}$ species can be best recovered using twochannel fitting most likely due to better statistics. For the case of substantial spectral overlap, the $\mathrm{O}$ species can be best recovered using 15-channel fitting to best remove overlap. In any case, the recovered diffusion times are within a factor of 1.5 of the expected value. It is also interesting to note that in all cases the recovered diffusion time shows a consistent bias from the expected value. This bias is observed to be strongly dependent on the number of fitting channels but independent of signal level. Since this bias is independent of signal level, it cannot be due to just random noise. This bias must originate from some inaccuracies in the assumptions of the global fitting algorithm. For example, the spectra of the individual species may not be perfectly determined. The origin of this bias will be best addressed in the future by globally fitting Monte Carlo simulation of experimental data.

We also demonstrate that combining a global fitting algorithm with our spectrally resolved FCS setup can effectively recover diffusion times in addition to number concentrations for complex mixtures of three species with overlapping spectra (Figure 4). The SRFCS with global fitting outperforms the typical three-channel detection scheme to recover the number concentrations of species with significant spectral overlap. Overall, the retrieved diffusion times for the independent three-channel data of the G, O, and R mixtures are typically consistent with expected results. In comparison, the SRFCS with global analysis method recovers diffusion coefficients for the brighter $\mathrm{G}$ species and the spectrally isolated $\mathrm{R}$ species with reasonable accuracy, but the recovery of the $\mathrm{O}$ is typically less accurate, which is likely due to spectral overlap (SI Figure 2).

The comparison between the 8-channel and the 15-channel results reveals that increased binning of the photon counts does not increase the accuracy of the recovered results (Figure 6). While the photon statistics for 8-channel data sets are improved with respect to 15channel data sets, resolving power of the instrumentation decreases. Thus, it is likely that the benefits of increased photon statistics are outweighed by the benefits of increased spectral resolution. When we included the spectral constraints in the SRFCS-G analysis, we did not see significant improvements in the accuracy of recovered diffusion times or concentration numbers, but we also did not observe an overall decrease in the recovery accuracy. Future experiments with multicolor complexes or complex mixtures of diffusing species with unique diffusion times may reveal that spectral constraints can improve the recovery accuracy of the SRFCS-G analysis.

With SRFCS-G, the major drawback that requires consideration with respect to this work is the low photon detection yield of this instrument. This SRFCS system is less sensitive than previously described multichannel FCS instruments due to the use of non-descanning 
geometry, loss at the grating spectrograph, and the use of lower quantum efficiency multianode PMT. We understand that the low detection yield limits the number of biological systems that can be studied although significant improvement of detection yield will be implemented in the future. First, a de-scanning detection scheme was employed in this microscope since we also use the same instrument for spectral resolved imaging. Since the use of non-descanning geometry is not necessary for single-point FCS measurement, better signal recovery can be achieved by placing the detection system in the non-descanned beam path. ${ }^{36}$ Second, the signal loss at the grating spectrograph can be eliminated by using an antireflection-coated prism instead. ${ }^{36}$ However, the lower dispersion efficiency of prisms as compared with gratings will require a large distance between the spectral dispersion element and the detector resulting in an instrument with larger physical size. Third, the multianode photomultiplier tube used in our study has very low quantum efficiency in the red portion of the spectrum. Today, new photocathode material has been introduced by the detector manufacturer and can significantly improve our sensitivity in the red. Some manufacturers are also experimenting with the fabrication of low-noise, high-sensitivity avalanche photodiode arrays. Another very promising technology is the newly commercially available multichannel hybrid photomultipliers. ${ }^{37,38}$ The availability of these higher sensitivity array detectors will make SRFCS-G a more competitive technology.

In comparison to a conventional FCS setup, the spectral detection yields a summed molecular brightness of Alexa 488 of $\sim 1.7 \mathrm{kHz} /$ molecule, which is $\sim 10$-fold less than a conventional FCS configuration using avalanche photodiodes for detection. While recovering number densities of molecules has proved to be difficult due to detection efficiency, improvements to the detection configuration, such as replacing the descanning optics and grating with a prism, will provide a 3 -fold increase in the detection efficiency. Combined with the 3 -fold increase in collection efficiency at $500 \mathrm{~nm}$ and over 25 -fold increase at $650 \mathrm{~nm}$ offered by implementing a multianode hybrid-PMT with new photocathode material, it should be possible to use this scheme to perform SRCS-G on single-molecule systems.

Although SRFCS-G is limited by the detection efficiency of this generation of instrument, the increased accuracy for recovering number density and diffusion coefficients of species with overlapping spectra support the claim that this approach has utility to future FCS applications that require the resolution of systems with multicolor constituents. Furthermore, the favorable comparison of recovered FCS parameters for 15-channel and binned 8-channel data suggests that the benefits of spectral resolution may outweigh the benefits of improved photon statistics in binning for some situations (Figure 6). The use of a global fitting approach with a parallel species detection scheme is an effective tool for multicolor FCS applications.

\section{CONCLUSIONS}

For a mixture of independent fluorophores with overlapping spectra, we show that the combination of a spectral-resolved detection system and global analysis improves upon standard FCS techniques for recovering accurate ratios of mixtures of fluorophores with overlapping spectra. The replacement of the grating spectrometer with a Brewster prism 
should also improve signal throughput. ${ }^{36}$ Further, future availability of higher sensitivity multichannel detectors will greatly enhance the utility of this approach in FCS. Our results suggest that multicolor FCS applications are not limited to the use of fluorescent species with well-resolved spectra. Thus, it is possible to expand the applicability of singlewavelength FCCS, whereby fluorophores with similar excitation characteristics and overlapping emission spectra also become candidates for use with this technique. ${ }^{12}$

\section{Supplementary Material}

Refer to Web version on PubMed Central for supplementary material.

\section{Acknowledgments}

This work has been supported by the NIH grant PO1HL64858.

\section{References}

1. Elson EL, Magde D. Biopolymers. 1974; 13:1-27.

2. Magde D, Elson EL, Webb WW. Biopolymers. 1974; 13:29-61. [PubMed: 4818131]

3. Thompson, NL. Topics in Fluorscence Spectroscopy, Techniques. 2nd. Lakowicz, J., editor. Vol. 1. Plenum Press; New York: 1991.

4. Hess ST, Huang SH, Heikal AA, Webb WW. Biochemistry. 2002; 41:697-705. [PubMed: 11790090]

5. Bacia K, Kim SA, Schwille P. Nat Methods. 2006; 3:83-89. [PubMed: 16432516]

6. Schwille P, MeyerAlmes FJ, Rigler R. Biophys J. 1997; 72:1878-1886. [PubMed: 9083691]

7. Heinze KG, Rarbach M, Jahnz M, Schwille P. Biophys J. 2002; 83:1671-1681. [PubMed: 12202390]

8. Heinze KG, Jahnz M, Schwille P. Biophys J. 2004; 86:506-516. [PubMed: 14695295]

9. Heinze KG, Koltermann A, Schwille P. Proc Natl Acad Sci USA. 2000; 97:10377-10382. [PubMed: 10973482]

10. Berland KM, So PTC, Gratton E. Biophys J. 1995; 68:694-701. [PubMed: 7696520]

11. Winkler T, Kettling U, Koltermann A, Eigen M. Proc Natl Acad Sci USA. 1999; 96:1375-1378. [PubMed: 9990031]

12. Hwang LC, Wohland T. Chemphyschem. 2004; 5:549-551. [PubMed: 15139229]

13. Heinze KG, Jahnz M, Schwilley P. Biophys J. 2004; 86:506-516. [PubMed: 14695295]

14. Schwille P. Cell Biochem Biophys. 2001; 34:383-408. [PubMed: 11898862]

15. Giepmans BNG, Adams SR, Ellisman MH, Tsien RY. Science. 2006; 312:217-224. [PubMed: 16614209]

16. Burkhardt M, Heinze KG, Schwille P. Opt Lett. 2005; 30:2266-2268. [PubMed: 16190439]

17. Müller JD, Chen Y, Gratton E. Biophys J. 2000; 78:474-486. [PubMed: 10620311]

18. Müller JD. Biophys J. 2004; 86:3981-3992. [PubMed: 15189894]

19. Ruttinger S, Macdonald R, Kramer B, Koberling F, Roos M, Hildt E. J Biomed Opt. 2006; 11

20. Lamb DC, Muller BK, Brauchle C. Curr Pharm Biotechnol. 2005; 6:405-414. [PubMed: 16248814]

21. Muller BK, Zaychikov E, Brauchle C, Lamb DC. Biophys J. 2005; 89:3508-3522. [PubMed: 16113120]

22. McHale K, Berglund AJ, Mabuchi H. Biophys J. 2004; 86:3409-3422. [PubMed: 15189843]

23. Haraguchi T, Shimi T, Koujin T, Hashiguchi N, Hiraoka Y. Genes Cells. 2002; 7:881-887. [PubMed: 12296819]

24. Lansford R, Bearman G, Fraser SE. J Biomed Opt. 2001; 6:311-318. [PubMed: 11516321] 
25. Zimmermann T. Microsc Tech. 2005; 95:245-265.

26. Skakun VV, Hink MA, Digris AV, Engel R, Novikov EG, Apanasovich VV, Visser A. Eur Biophys J Biophys Lett. 2005; 34:323-334.

27. Digris AV, Skakoun VV, Novikov EG, Apanasovich VV, Hink MA, Visser A. Biophys J. 2000; 78:440A-440A.

28. Eggeling C, Kask P, Winkler D, Jager S. Biophys J. 2005; 89:605-618. [PubMed: 15849243]

29. Weisshart K, Jungel V, Briddon SJ. Curr Pharm Biotechnol. 2004; 5:135-154. [PubMed: 15078148]

30. Buehler C, Kim KH, Greuter U, Schlumpf N, So PTC. J Fluoresc. 2005; 15:41-51. [PubMed: 15711876]

31. Beechem JM. Methods Enzymol. 1992; 210:37-54. [PubMed: 1584042]

32. Verveer PJ, Squire A, Bastiaens PIH. Biophys J. 2000; 78:2127-2137. [PubMed: 10733990]

33. Pelet S, Previte MJR, Laiho LH, So PTC. Biophys J. 2004; 87:2807-2817. [PubMed: 15454472]

34. Palo K, Metz U, Jager S, Kask P, Gall K. Biophys J. 2000; 79:2858-2866. [PubMed: 11106594]

35. Huber, PJ. Robust Statistics. Wiley; New York: 1981.

36. Hwang LC, Leutenegger M, Gosch M, Lasser T, Rigler P, Meier W, Wohland T. Opt Lett. 2006; 31:1310-1312. [PubMed: 16642095]

37. Suyama A, Fukasawa A, Haba J, Iijima T, Iwata S, Sakuda M, Sumiyoshi T, Takasaki E, Tanaka M, Tsuboyama T, Yamada Y. IEEE Trans Nucl Sci. 2004; 51:1056-1059.

38. Suyama M, Fukasawa A, Haba J, Iijima T, Iwata S, Sakuda M, Sumiyoshi T, Takasaki F, Tanaka M, Tsuboyama T, Yamada Yoshikazu. Nucl Instr Methods Phys Res, Sect A. 2004; 523:147-157. 


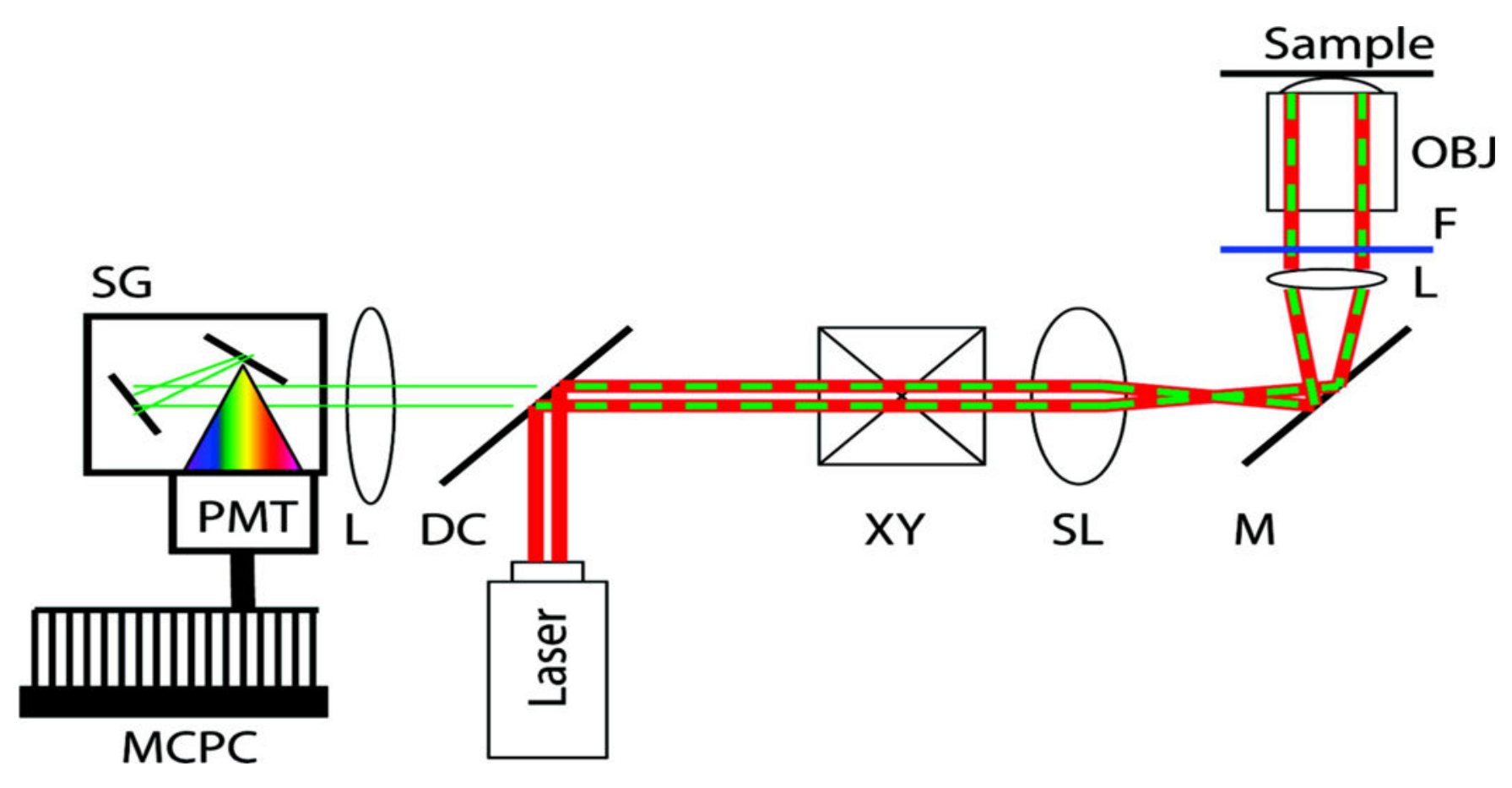

Figure 1.

Multicolor FCS system combining a two-photon scanning microscope, a multianode PMT, and a multichannel single-photon counting electronics (MCPC) (galvonmeter scanning mirrors (XY); scan lens (SL); lens (L); silver mirror (M); BG39 filter (F); a dichroic mirror (DC); grating spectrograph (SG)). 

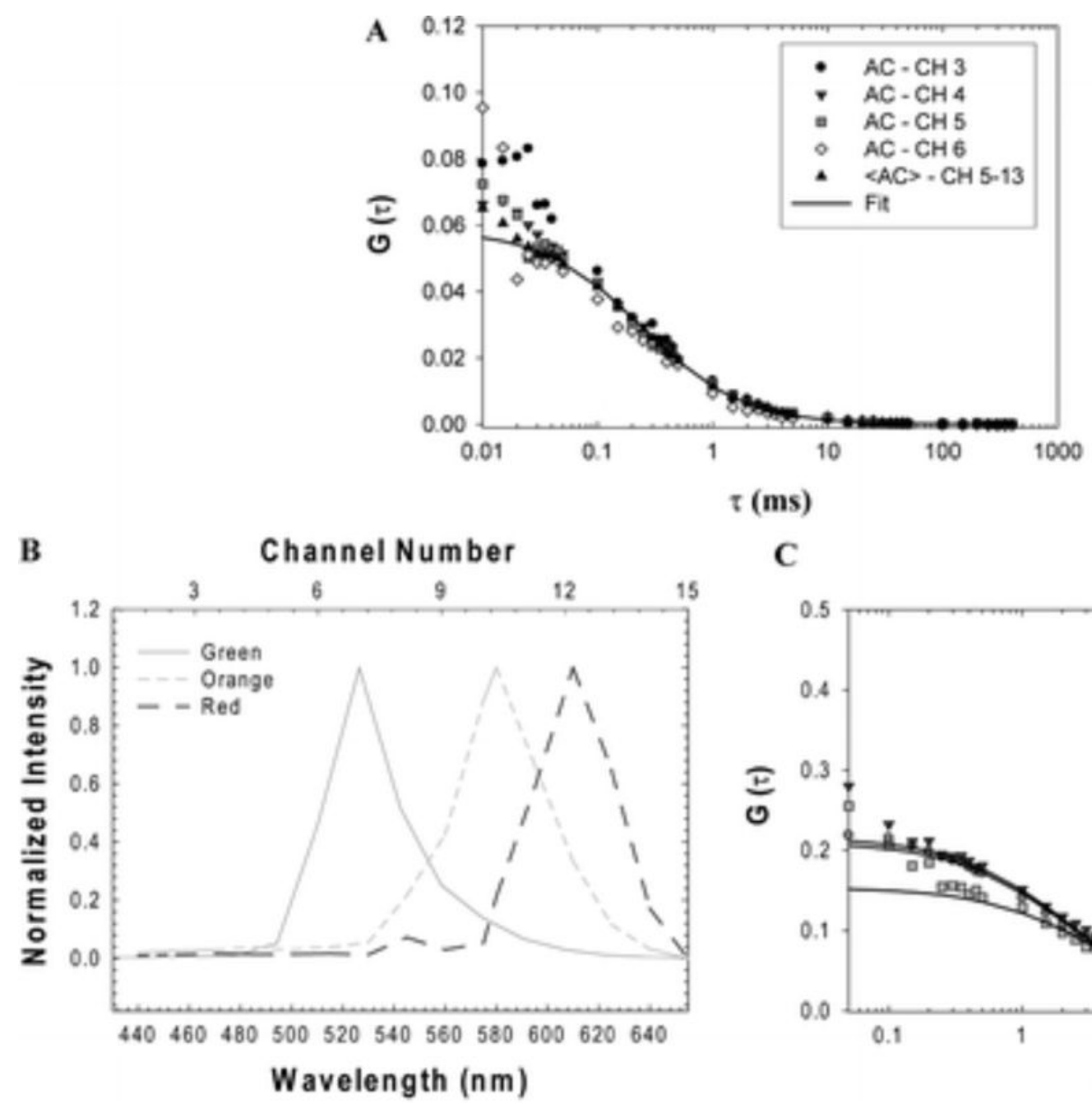

C

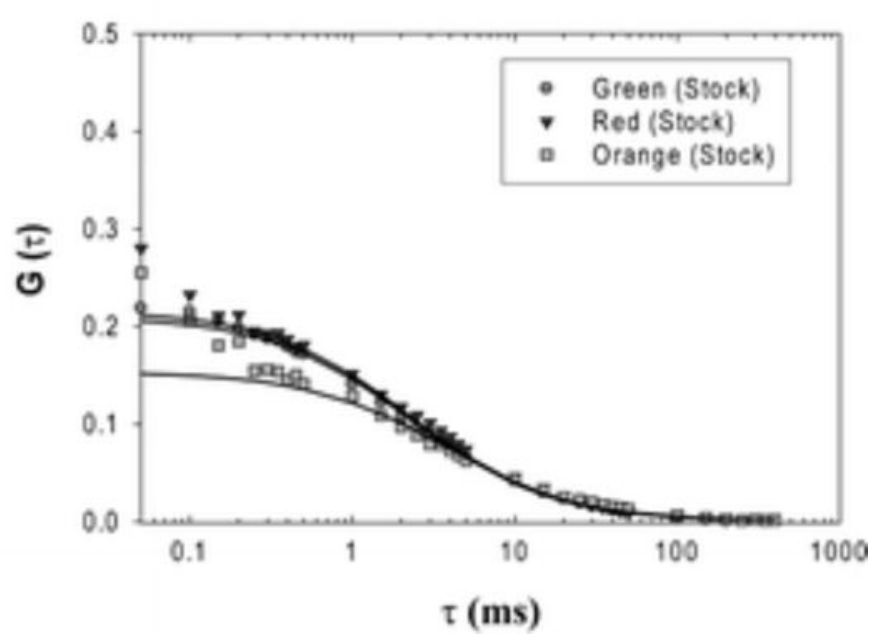

Figure 2.

(A) Autocorrelation curves of channels 3-6 and the average for spectral detection of $25 \mathrm{nM}$ solution of Alexa488 in a 50\% glycerol aqueous solution. Single-channel correlation fit is also shown (solid line). (B) Normalized intensity spectra for green $(\mathrm{G})$, orange $(\mathrm{O})$, and red (R) 20-nm-diameter beads plotted versus wavelength and corresponding channel number. (C) Single-channel autocorrelation curves for stock solutions of green, orange, and red beads. 

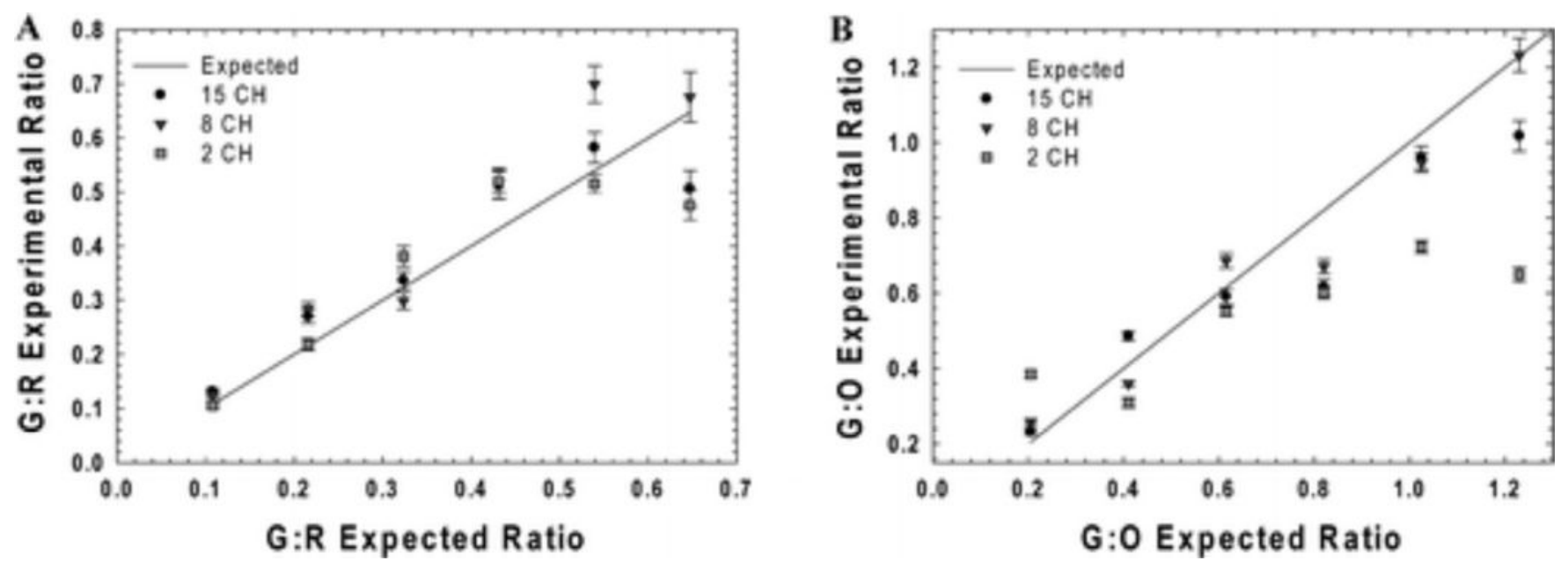

Figure 3.

(A) Recovered experimental ratios of two-color mixtures with non-overlapping spectra of green $(\mathrm{G})$ and red (R) 20-nm beads. (B) Recovered experimental ratios of two-color mixtures with spectral overlap of green $(\mathrm{G})$ and orange $(\mathrm{O}) 20$-nm beads. Data are fit using 15-channel SRFCS with global analysis (15 CH, circles); 8-channel SRFCS with global analysis ( $8 \mathrm{CH}$, inverted triangles); and least-square fitting of the autocorrelation curves from binned $\mathrm{G}$ and $\mathrm{R}$ or $\mathrm{G}$ and $\mathrm{O}$ data ( $2 \mathrm{CH}$, squares). Expected recovered ratios are shown for reference (expected, solid line). 

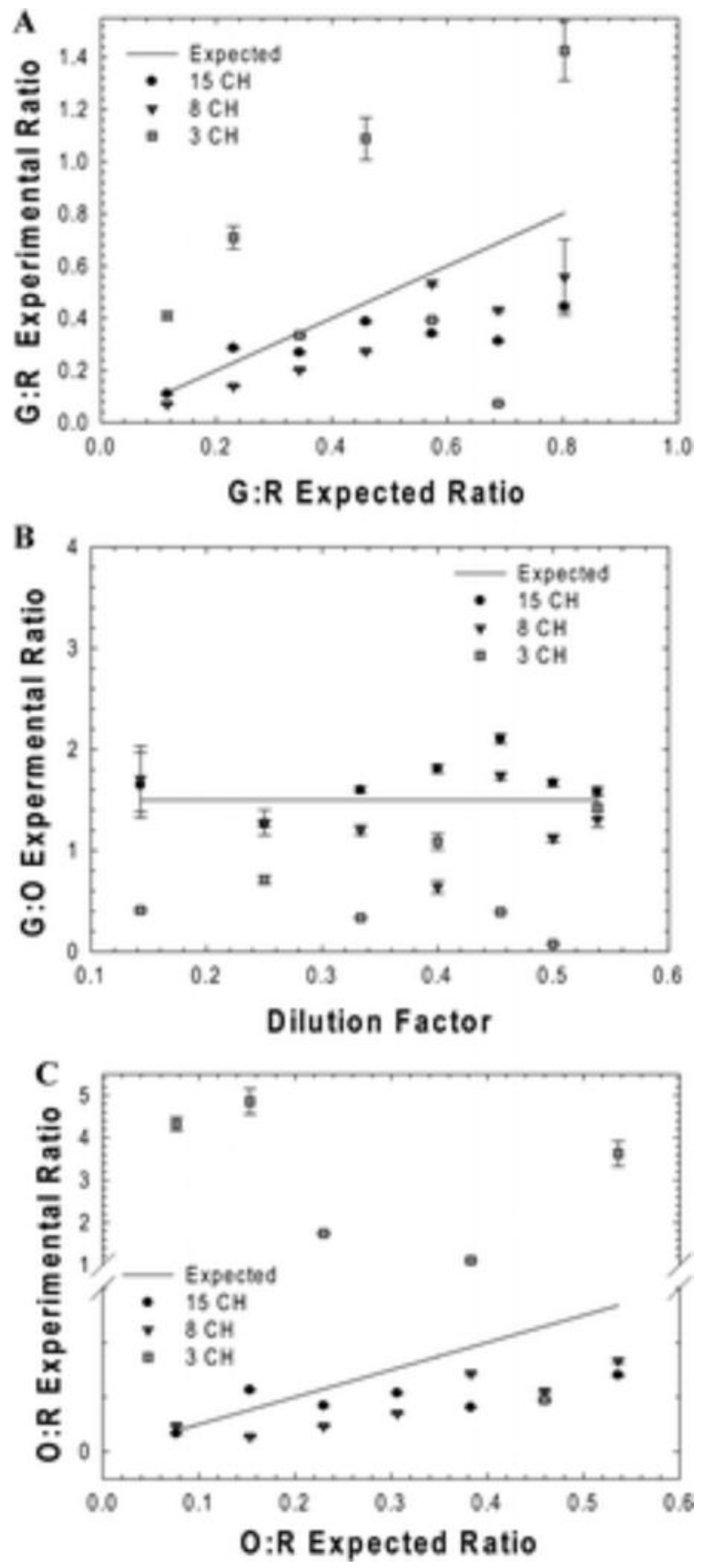

Figure 4.

Recovered experimental ratios of three-color mixtures of green $(\mathrm{G})$, orange $(\mathrm{O})$, and red $(\mathrm{R})$ 20-nm beads: (A) Recovered G/R experimental ratio. (B) Recovered G/O experimental ratio. (C) Recovered O/R experimental ratio. Data are fit using 15-channel SRFCS with global analysis (15 CH, circles), 8-channel SRFCS with global analysis with ( $8 \mathrm{CH}$, inverted triangles), and least-squares fitting of the autocorrelation curves from binned $\mathrm{G}, \mathrm{R}$, and $\mathrm{O}$ data (3 $\mathrm{CH}$, squares). Expected recovered ratios are shown for reference (expected, solid line). Since $\mathrm{G} / \mathrm{O}$ ratios are constant, the $\mathrm{G} / \mathrm{O}$ recovered ratios were plotted versus dilution factor. 

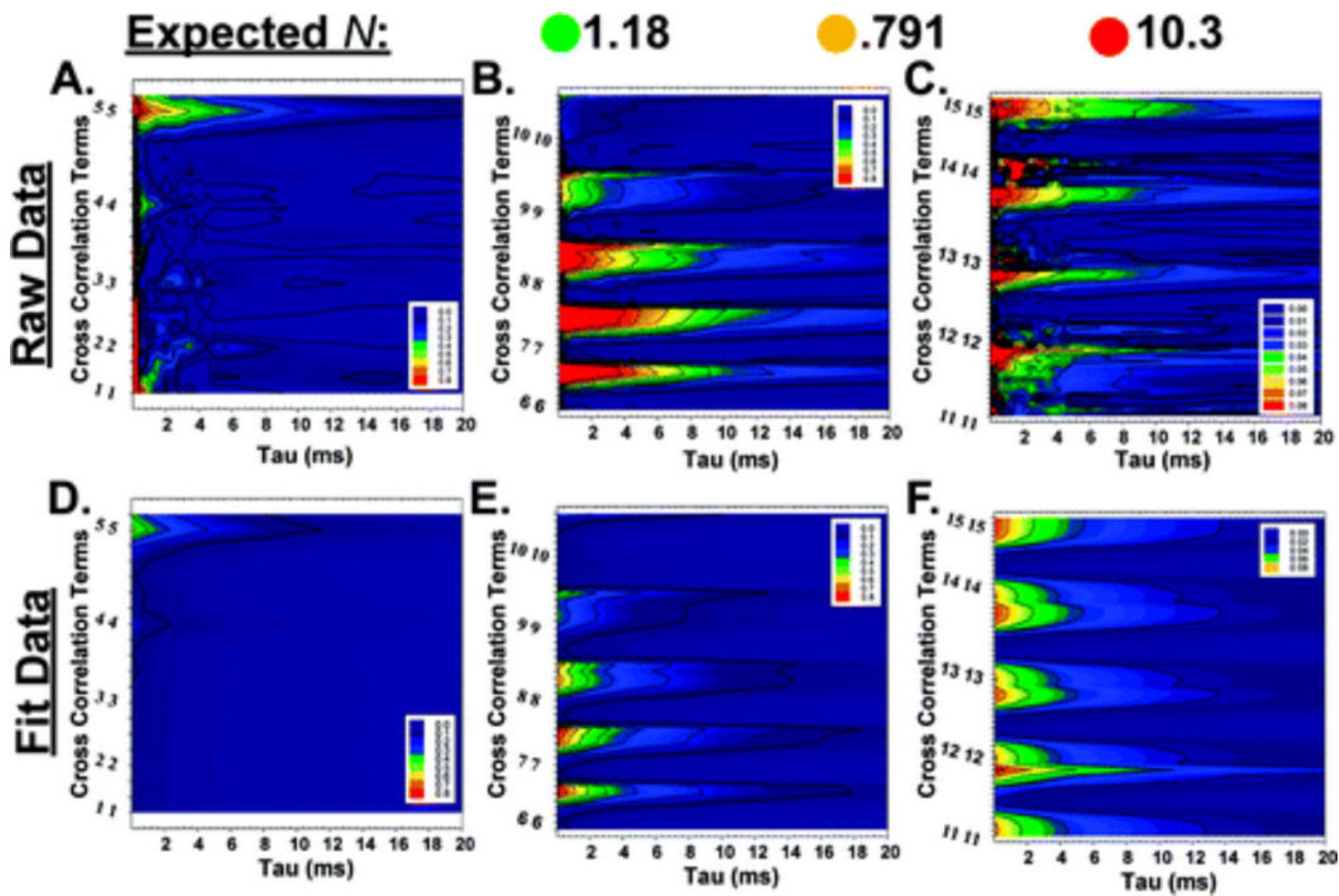

Figure 5.

Raw data surface plots of a three-color mixture of beads that contains approximately 1.18 green beads, 0.791 orange beads, and 10.3 red beads. Cross-correlation decays are shown for channels 1-5 in (A), 76-10 in (B), and 11-15 in (C). Contour plots of the fit data from the same three-color mixture. Cross-correlation decays are shown for channels 1-5 in (D), 6-10 in (E), and-11-15 in (F). The legend displays the color scale for the $\mathrm{G}(0)$ amplitudes ( $z$-axis data) and the $y$-axis specifies the specific cross-correlation term (see text for further description). 


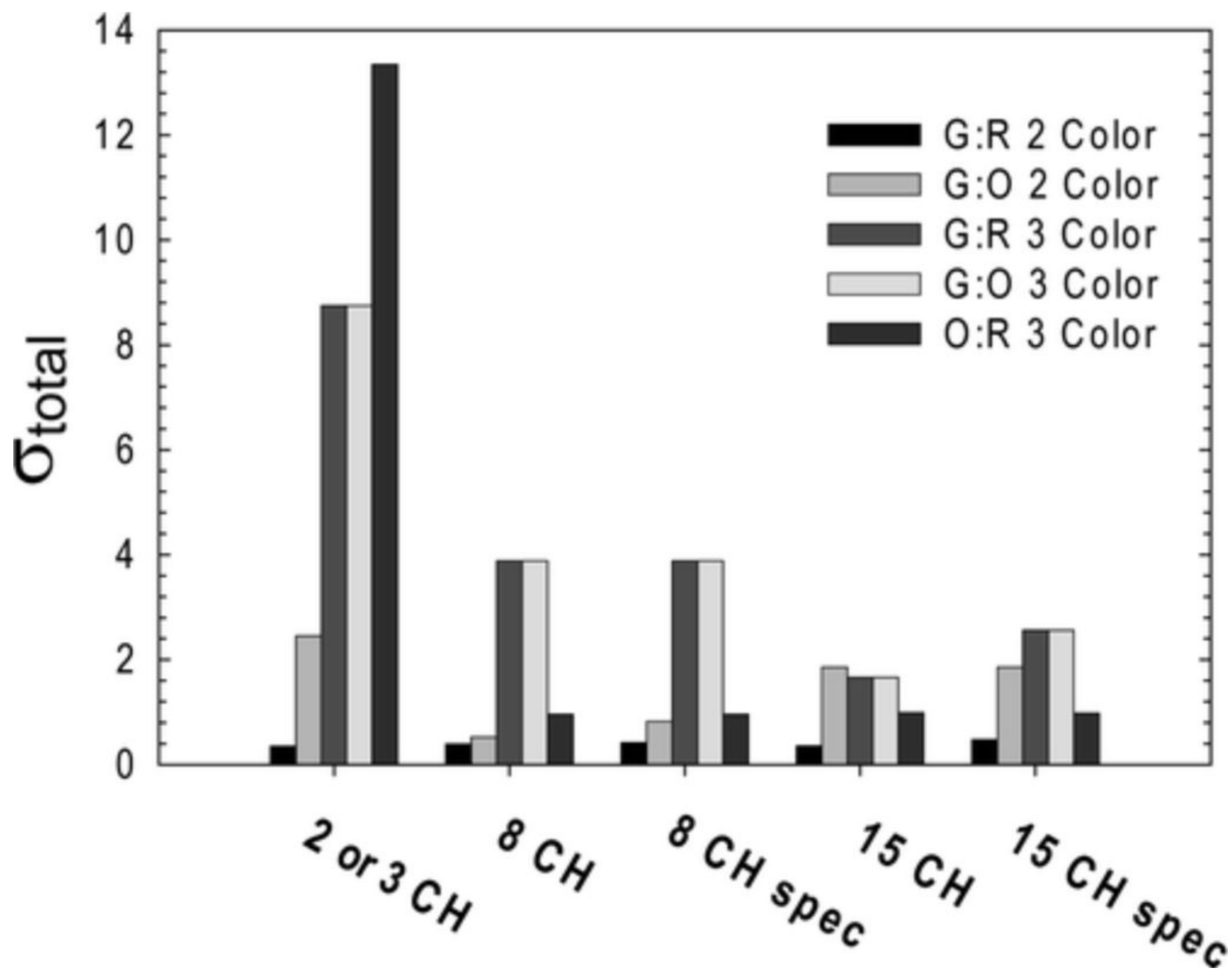

Figure 6.

Overall deviations of the number densities of all mixtures, $n$, in the two-color and threecolor experiments. Two- and three-color results for $\mathrm{G}$ and $\mathrm{R}, \mathrm{G}$ and $\mathrm{O}$ data, or $\mathrm{O}$ and $\mathrm{R}$ data are shown for least-squares fitting of the autocorrelation curves from binned 2- or 3-channel data $(2$ or $3 \mathrm{CH})$; 8-channel SRFCS with global analysis without $(8 \mathrm{CH})$ and with spectral constraints (8 CH spec); and 15-channel SRFCS with global analysis without $(15 \mathrm{CH})$ and with spectral constraints (15 $\mathrm{CH}$ spec). 